УДК 616.34-005.1; 617.553; 616-089-06

DOI

\title{
ВТОРИЧНЫЙ АОРТО-ДУОДЕНАЛЬНЫЙ СВИЩ. РЕЦИДИВИРУЮЩЕЕ КРОВОТЕЧЕНИЕ. ТРУДНОСТИ ТОПИЧЕСКОЙ ДИАГНОСТИКИ И ЛЕЧЕБНО- ТАКТИЧЕСКИЕ ОШИБКИ (СЛУЧАЙ ИЗ КЛИНИЧЕСКОЙ ПРАКТИКИ)
}

\section{SECONDARY AORTO-DUODENAL FISTULA. RECURRENT BLEEDING. DIFFICULTIES OF TOPICAL DIAGNOSIS AND TACTICAL ERRORS (CASE FROM CLINICAL PRACTICE)}

\author{
А.И. Панасюк ${ }^{1,2}$, С.А. Кыштымов ${ }^{1,2}$, Е.О. Иноземцев ${ }^{2}$, Е.Г. Григорьев ${ }^{1}$ \\ A.I. Panasyuk ${ }^{1,2}$, S.A. Kyshtymov ${ }^{1,2}$, E.O. Inozemcev ${ }^{2}$, E.G. Grigoryev ${ }^{1}$ \\ ${ }^{1}$ Иркутский государственный медицинский университет, \\ Россия, 664049, Иркутск, ул. Красного Восстания, д. 1 \\ ${ }^{2}$ Иркутская областная клиническая больница, \\ Россия, 664049, Иркутск, мкр. Юбилейный, д. 100 \\ ${ }^{1}$ Irkutsk State Medical University, \\ 1 Krasnogo Vosstaniya St., 664003, Irkutsk, Russia \\ ${ }^{2}$ Irkutsk Regional Hospital, \\ 100 Yubileyniy district, 664049, Irkutsk, Russia \\ E-mail: 77Eugene@rambler.ru
}

\begin{abstract}
Аннотация
В статье представлено наблюдение вторичного аорто-дигестивного свища, возникшего через 8 лет после протезирования аорты. Приведена статистика развития первичных и вторичных аортодигестивных свищей, причины их возникновения, локализации, основные клинические симптомы и методы диагностики. Обсуждаются различные лечебно-тактические подходы. На конкретном клиническом наблюдении демонстрируется случай протезирования аорты в условиях инфекционного процесса, осложнившийся развитием вторичного аорто-дигестивного свища спустя 8 лет. Обсуждается сложность диагностики и лечения данной группы пациентов. Отдельно делается акцент на лечебно-тактических ошибках.
\end{abstract}

\begin{abstract}
The paper presents the case report of a secondary aorto-digestive fistula, which developed eight years after aortic prosthetics and was complicated by recurrent hemorrhage and anemia. The authors review the incidence of primary and secondary aorto-digestive fistulas, causes of their occurrence, localization variants, main clinical symptoms and methods of instrumental diagnostics. Various therapeutic and tactical approaches are discussed. With specific reference to the clinical case, the authors explain the complexity of topical diagnosis of the hemorrhage origin and treatment of this group of patients. Special attention is paid to therapeutic and tactical faults due to the lack of accurate information about the localization of aorto-digestive fistula. The complexity of the situation was also accounted for the effects of aortic prosthesis performed under retroperitoneal suppurative process. The article interprets the reasons for inadequate information value of MSCT-aortography and invasive aortography.
\end{abstract}

Ключевые слова: протезирование аорты, забрюшинный абсцесс, аорто-дуоденальная фистула, желудочно-кишечное кровотечение.

Keywords: aortic prosthetics, retroperitoneal abscess, aorto-duodenal fistula, gastrointestinal bleeding. splenomegaly. 


\section{Введение}

Желудочно-кишечные кровотечения (ЖКК) одна из основных причин экстренной госпитализации в хирургический стационар. По этиологии ЖКК можно разделить на две почти равные группы: язвенной и не язвенной природы [Савельев, 2005]. Этапы оказания помощи пациентам с кровоточащими язвами желудка и двенадцатиперстной кишки (ДПК) хорошо разработаны и стандартизированы [Лебедев, Климов, 2010]. Кровотечения не язвенной природы включают в себя большое количество нозологических форм. Одной из наиболее редких причин ЖКК является аорто-дигестивный свищ (АДС) [Савельев, 2005].

Первичный свищ образуется вследствие осложненного течения аневризмы брюшного отдела аорты (расслоение, разрыв) и встречается в 0.04-0.07\% [Šumskienè et al., 2016]. Чаще опорожнение аневризмы происходит в просвет ДПК $(81,6 \%)$, сообщение аорты с тощей кишкой описано в $8 \%$ наблюдений, толстой - в 5,5\% и желудком - в 4,9\% [Покровский, 1979]. Вторичный свищ, возникающий после протезирования аорты, встречается чаще $(0,5-2,3 \%)$ [Šumskienė et al., 2016]. Основным клиническим симптомом АДС является внезапная боль в животе, позже появляются рвота, кал, окрашенный кровью, коллапс и анемия [Покровский, 1979].

Неотложное хирургическое вмешательство заключается в протезировании или репротезировании аорты и ушивании дефекта полого органа. Летальность достигает $90 \%$ [Lirici et al., 2019].

\section{Клиническое наблюдение}

Пациент, 58 лет, поступил в Иркутскую областную клиническую больницу 19.04.2019 через 4 суток после первого эпизода желудочно-кишечного кровотечения. Из анамнеза известно, что в 1996 году выполнена левосторонняя нефрэктомия по поводу вторично-сморщенной подковообразной почки. 08.06.2011 поступил в отделение урологии с жалобами на боли в поясничной области слева. Выявлено жидкостное образование в ложе удаленной почки. Вскрыта полость, содержащая сгустки крови, после удаления которых открылось артериальное кровотечение. Тампонирование полости гематомы. Выполнена селективная артериография: ложная аневризма аорты на уровне культи левой почечной артерии. После лапаротомии вскрыт забрюшинный абсцесс до 2 мл, на уровне почечных артерий. Обнаружен дефект в стенке аорты до 0,7 см, ушит узловыми швами. После удаления тампонов установлен линейный разрыв аорты перед устьем правой почечной артерии. Артерия отсечена от аорты, дефект ушит. Восстановление почечного артериального кровотока протезом Impra Carboflo REF 10S07C с вшиванием его в аорту. Время окклюзии 45 минут. Санация, дренирование брюшной полости, забрюшинного пространства. Течение послеоперационного периода тяжелое. С 10.06.2011 появились жалобы на боли в правой нижней конечности. Пульс на бедренной артерии не определялся, чувствительность, движения сохранялись. МСКТ диагностировала тромбоз правой общей подвздошной артерии на фоне тотального кальциноза. Проведено рентгенохирургическое стентирование. Выписан 05.07.2011.

Повторно поступил 13.07.2011 с клиникой абсцесса забрюшинного пространства, диагноз подтверждён МСКТ. Проекционным доступом в левой пояснично-боковой области живота вскрыта инфицированная гематома (Klebsiella pneumonia, Pseudomonas aeruginosa, Serratia marcescens $-10^{3}$ ). Через сутки в связи с клиникой геморрагического шока проведена аортография - разрыв аневризмы брюшной аорты с формированием забрюшинной гематомы. Выполнена лапаротомия. В брюшной полости выраженный спаечный процесс. Через правую бедренную артерию в аорту заведен окклюзионный баллон - гемостаз. Причина кровотечения - дефект в устье правой общей подвздошной артерии, через который пролабирует стент. Аорта пересечена на 3 см выше бифуркации, дистальный конец ушит. Аорто-бифеморальное протезирование (Intervascular IGK2010s.). Забрюшинная гематома опорожнена - 200 мл сгустков. К аортальному анастомозу установлены гемоста- 
тические тампоны. 17.07.2011 выполнены запланированная релапаротомия, ревизия протеза, энтеролиз, санация, дренирование брюшной полости и забрюшинного пространства. Выписан 27.09.2011 в удовлетворительном состоянии. В течение 8 лет наблюдался по месту жительства.

При поступлении 19.04.2019 состояние средней тяжести. В сознании, контакту доступен. Кожа бледная. Пульс 78 в минуту, давление 100/60 мм рт. ст. При пальпации передняя брюшная стенка не напряжена. Аускультативно сосудистых шумов нет, перистальтика кишечника выслушивается. Пульсация брюшного отдела аорты определяется. Пальцевое исследование прямой кишки безболезненное, тонус сфинктеров сохранен, ампула свободна, на перчатке кал черного цвета. ОАК: гемоглобин 78 г/л, эритроцитов 2,8×109, лейкоцитов 7,54×109, тромбоцитов $311 \times 10^{9}$.

Учитывая анамнез, заподозрено развитие АДС. Выполнена МСКТ-ангиография (рис. $1,2)$. Выявлены послеоперационные изменения в брюшной полости: аорта, протез отчетливо визуализируются, данных за наличие АДС нет. При ФГДС органической патологии пищевода, желудка и ДПК не обнаружено. При колоноскопии выявлены полипы толстой кишки 0,3-0,7 см без признаков кровотечения, увеличенные наружные и внутренние геморроидальные узлы (эрозированные, с мелкими тромбами).

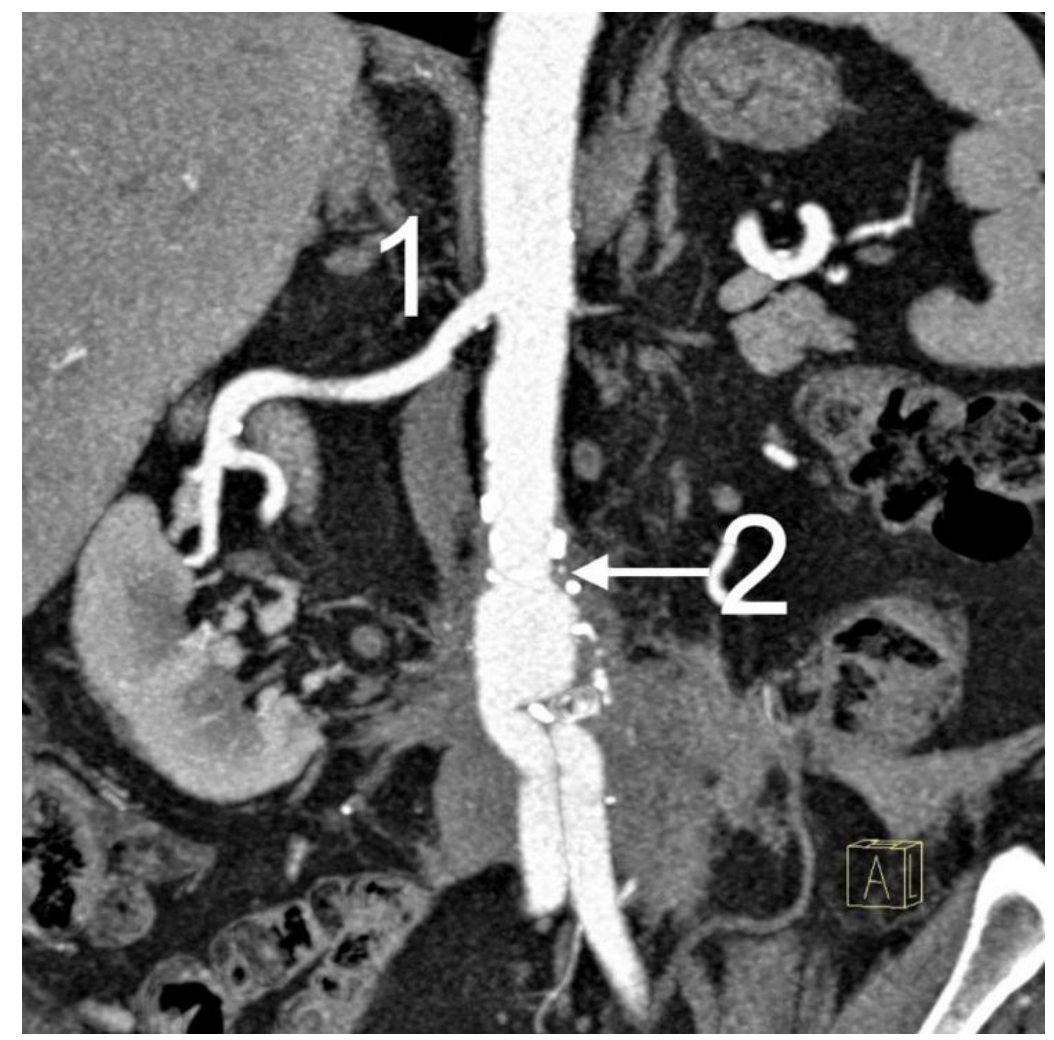

Рис. 1. МСКТ-ангиография брюшного отдела аорты. 1 - протез правой почечной артерии, кальциноз аорты; 2 - проксимальный анастомоз. Уклонения контрастированной крови за пределы сосудистого русла нет

Fig. 1. MSCT-angiography of the abdominal aorta. 1 - prosthesis of the right renal artery, calcification of the aorta; 2 - proximal anastomosis. Leakage of contrasted blood outside the vascular bed is absent

Решено, что возможной причиной анемизирующего кровотечения является осложненная геморроидальная болезнь (безусловная ошибка), в связи с чем 23.04.2019 выполнена геморроидэктомия по Миллигану - Моргану. Гладкое течение послеоперационного периода. На фоне полного благополучия 30.04 .2019 развилась клиника массивного желудочно-кишечного кровотечения: коллапс, бледность кожи и слизистых оболочек. На ФГДС - желчь в желудке и ДПК, крови нет. 


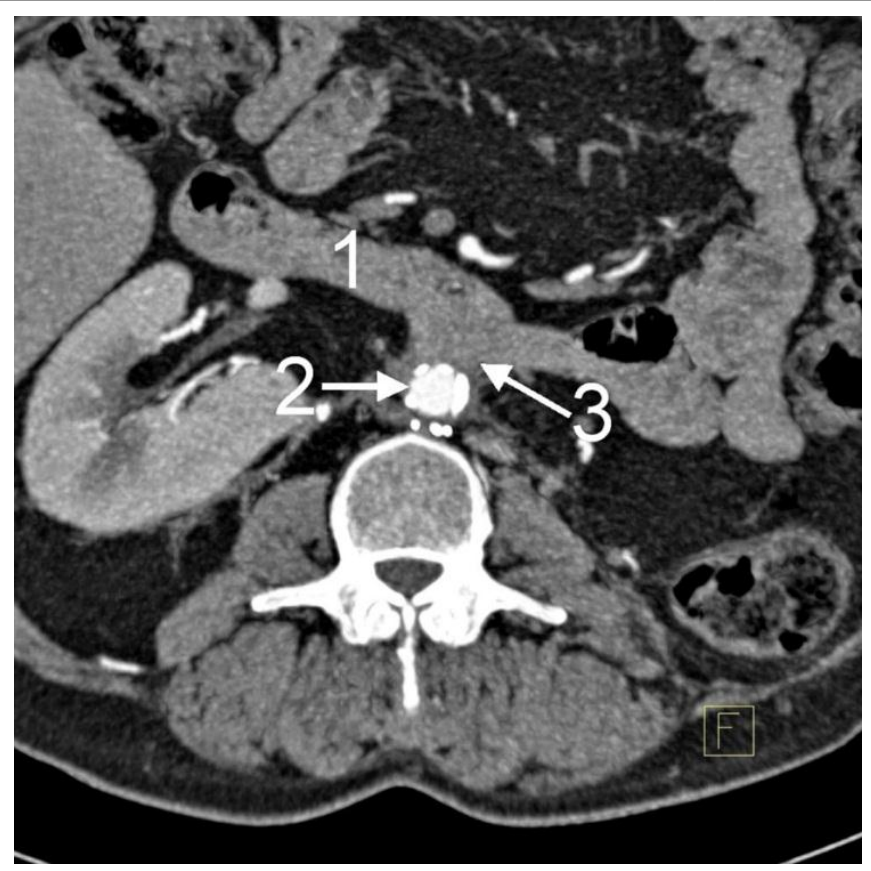

Рис. 2. МСКТ-ангиография. 1 - нижняя горизонтальная ветвь ДПК; 2 - аорта с кальцинозом стенки; 3 - перивазальные тромботические массы. Уклонения контраста нет. Место наиболее вероятного нахождения свища

Fig. 2. MSCT-angiography. 1 - The lower horizontal branch of the duodenum; 2 - aorta with the wall calcification; 3 - perivasal thrombotic masses. Contrast leakage is absent. The most probable fistula location

Выполнена инвазивная аортография, экстравазации контрастированной крови нет (рис. 3). Проводилась консервативная терапия, переливание компонентов крови.

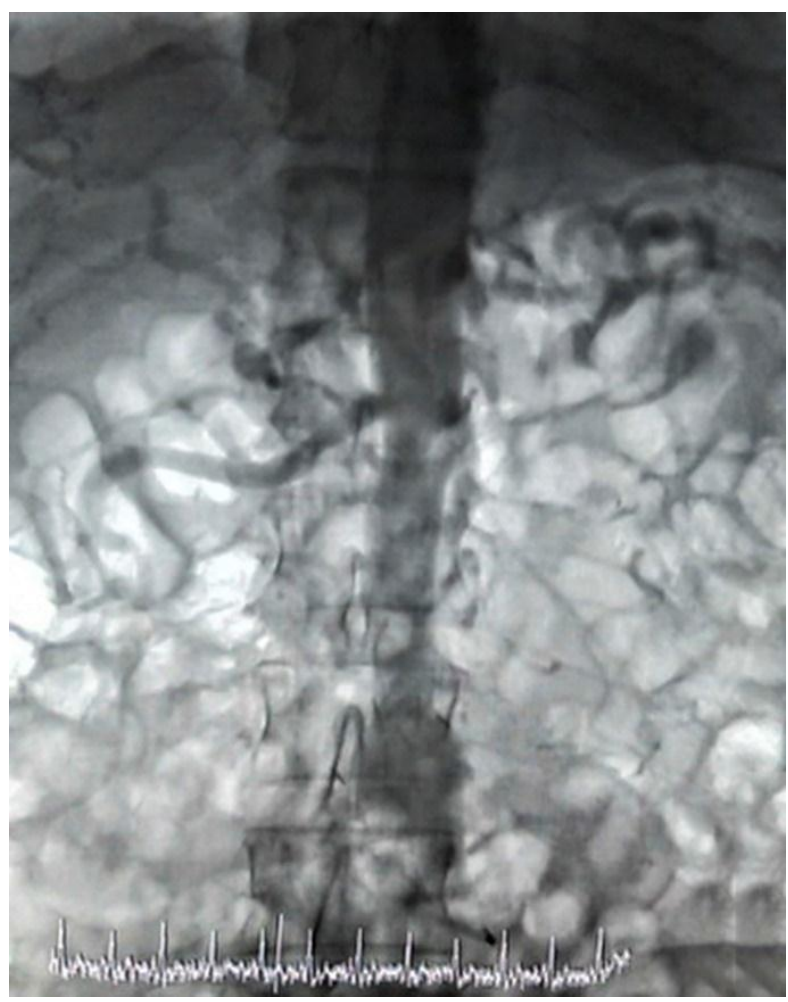

Рис. 3. Инвазивная аортография (описание в тексте)

Fig. 3. Invasive aortography (description in text) 
Очередной эпизод кровотечения случился 05.05.2019. По жизненным показаниям выполнена лапаротомия. Брюшная полость облитерирована. Длительный энтеролиз. В подвздошной, сигмовидной кишках кровь. Тощая кишка дистальнее дуоденоеюнального перехода на протяжении 1,5 м плотно фиксирована рубцом к аорте, протезу. Интраоперационная дуоденоскопия патологии не выявила. Тощая кишка отделена от протеза, вскрылся абсцесс (10 мл густого серого гноя без запаха - Pseudomonas aeruginosa $\left.10^{4}\right)$. Стенка в этом месте отечна, инфильтрирована - вероятный источник кровотечения? Выполнена резекция 3/4 по А.В. Мельникову (очередная ошибка). Слизистая оболочка ярко гиперемирована, кровоточащего сосуда нет. Кишка ушита отдельными внеслизистыми швами. Выполнена назоинтестинальная интубация. Одной из стенок опорожненного гнойника является сосудистый протез. Кровотечения нет, забрюшинно полость распространяется к мезосигме. Назначена санационная релапаротомия через 24 часа.

При повторном осмотре брюшной полости установлено, что тонкая кишка отечная, швы состоятельны. В её просвете следы крови. Интраоперационная ФКС патологии ободочной кишки не обнаружила. К левой бранше аорто-подвздошного протеза плотно фиксирована сигмовидная кишка. При мобилизации вскрылся её просвет на протяжении 15 см. Выполнена вынужденная левосторонняя гемиколэктомия, трансверзостомия. Программированная релапаротомия через 24 часа.

При очередной ревизии живота в брюшной полости обнаружено умеренное количество серозного выпота. Петли кишечника не дилатированы. Удалены тампоны из брюшной полости. Кровотечения нет. Санация, дренирование.

11.05.2019 рецидив ЖКК. На гастроскопии обнаружены острые эрозии слизистой оболочки желудка с признаками состоявшегося кровотечения. Назначена антисекреторная, гемостатическая терапия, гемотрансфузии, переливание свежезамороженной плазмы. 14.05.2019 вновь развилось желудочное кровотечение. На гастроскопии в дне желудка диагностирована язва Дьелафуа (?), выполнено клипирование сосуда. Очередной эпизод кровотечения возник через 10 дней. Выполнена аортография, все фазы контрастирования наступают своевременно, экстравазации нет. Продолжено консервативное лечение, состояние стабилизировано.

04.06.2019 вновь эпизод кровотечения. Выполнена ФГДС, просвет ДПК на всем протяжении заполнен кровью. Продолжающегося кровотечения нет.

Выполнена лапаротомия. Тампонада кровью желудка и ДПК. С техническими трудностями выделен дуоденоеюнальный переход. Дуоденотомия. Удалено до 1 литра сгустков крови. Началось артериальное кровотечение. Пальцевое прижатие. С техническими трудностями через просвет кишки удалось визуализировать аорто-двенадцатиперстный свищ до 0,5 см, на задней стенке нижней горизонтальной ветви ДПК. Виден протез и полипропиленовые швы (рис. 4). Удалось ушить дефект проленом 3/0. Учитывая тяжесть состояния, операция завершена тампонированием забрюшинного пространства.

\section{Обсуждение}

Основными причинами развития вторичного аорто-дигестивного свища являются механическое воздействие протеза на стенку кишки (пролежень), усиливающееся за счет пульсовой волны, и инфицирование трансплантата. В приведенном наблюдении основной причиной развития свища был длительный инфекционный процесс. Протезирование аорты по жизненным показаниям выполнялось в условиях забрюшинной флегмоны. Временной промежуток между этими событиями (протезирование - свищ) составил 8 лет.

Оптимальным методом диагностики АДС является МСКТ-ангиография [Романович и др., 2016; Трушникова и др., 2018], однако в обсуждаемом наблюдении признаков аортодуоденального сообщения не обнаружено. Несмотря на то, что анамнез известен, целенаправленный поиск АДС, включая инвазивную аортографию, длительное время был безуспешным. 


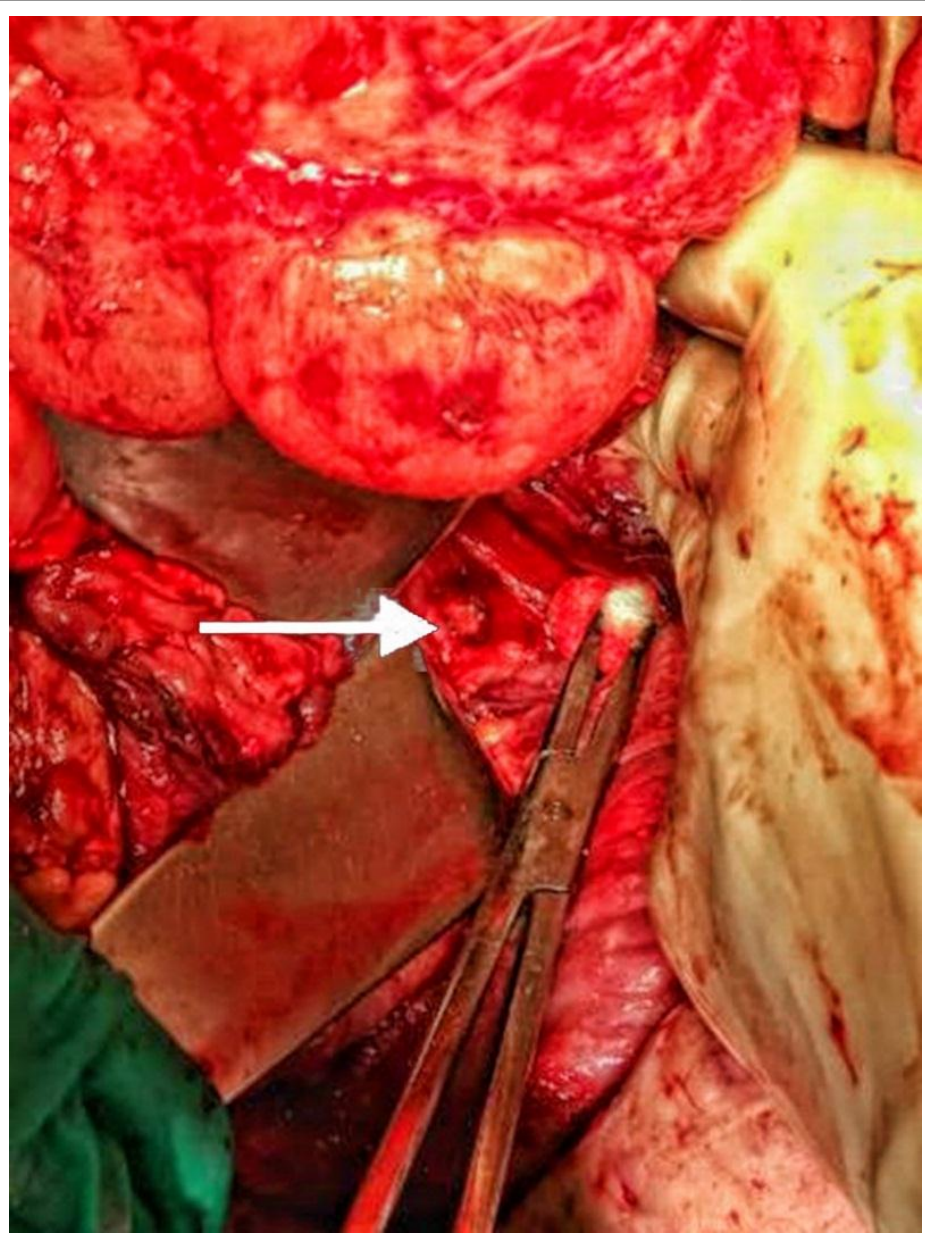

Рис. 4. Интраоперационное фото. После выполнения энтеротомии виден участок аорты со свищом, открывающимся в просвет кишки (стрелка)

Fig. 4. Intraoperative photo. After enterotomy, the aorta segment with a fistula opening into the intestinal lumen is visible (arrow)

На фоне нарастания полиорганной недостаточности наступил летальный исход.

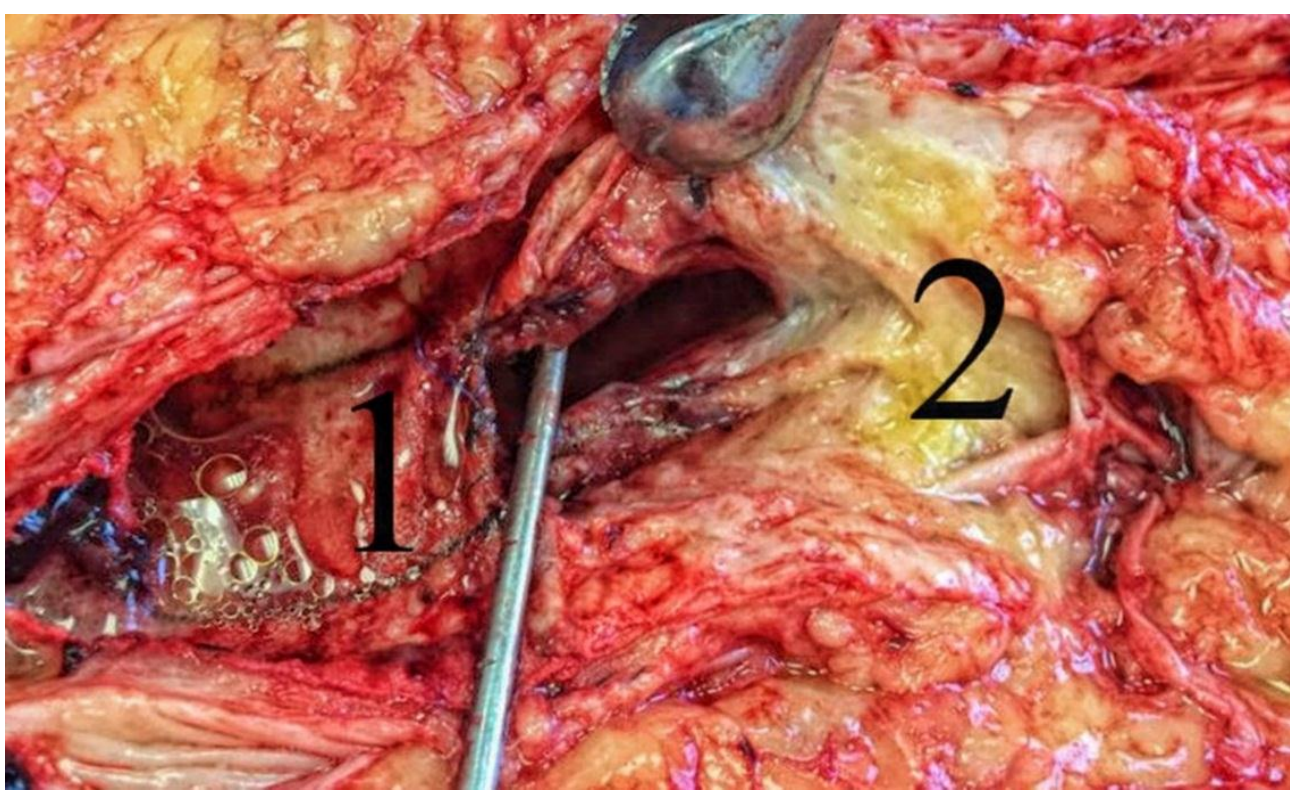

Рис. 5. Аутопсия. Вид со стороны просвета аорты. Зонд введен в свищ, открывающийся в просвет кишки Fig. 5. Autopsy. View from the aortic lumen. The probe is inserted into the fistula opening into the intestinal lumen 
При лапаротомиях выраженный рубцовый процесс не позволил обеспечить доступ к аортальному протезу, несмотря на достаточный опыт в лечении осложненной спаечной болезни, послеоперационных несформированных кишечных свищей.

Как отмечалось рядом авторов, при аорто-кишечном свище первое ЖКК, как правило, не бывает смертельным. Это связано с тем, что при гипотонии за счет перивазального тромбоза возможна временная остановка кровотечения [Покровский, 1979]. Стоит отметить, что в представленном наблюдении рецидив наступал после активизации пациента. При стабилизации гемодинамики происходил массивный сброс крови в просвет ДПК, после этого давление в аорте снижалось, формировались экстравазальные тромбы и кровотечение прекращалось. На МСКТ-ангиографии экстравазации контрастированной крови не обнаруживали. При ретроспективном анализе МСКТ на одном из срезов (см. рис. 2) вероятная локализация аортодуоденального свища установлена. Она соответствует интраоперационной находке.

Оптимальное лечение аорто-дигестивного свища активно обсуждается в литературе. При возникновении первичного АДС возможно протезирование аорты [Костоглодова и др., 2016; Куликовский и др., 2017,], вторичного - репротезирование, которое сопряжено с техническими трудностями - выраженным адгезивным процессом в зоне бывшего инфицирования. Во время разобщения АДС высока вероятность профузного кровотечения. Батвинков Н.И. первым этапом выполнял наложение подключично-бедренного анастомоза для сохранения единственной нижней конечности у пациента с АДС, вторым - удаление протеза инфраренального отдела аорты [Батвинков и др., 2013]. В качестве протеза аорты в случае успешного репротезирования Романович А.В. с коллегами использовали нативный аорто-бифеморальный комплекс, полученный от донора с констатированной смертью мозга [Романович и др., 2016]. Возможно использование протезов, импрегнированных серебром, с повышенной микроборезистентностью [Андрейчук и др., 2015].

Применяется этапное лечение аорто-дигестивного свища: выполняется эндоваскулярное протезирование, стентирование аорты графтом или внутриаортальная баллонная окклюзия с последующим репротезированием 131116 [Miyamoto et al., 2016; Colombi et al., 2019; Lirici et al., 2019]. В нашем случае применение данных методик было невозможно, так как уровень свища не был установлен. Ретроспективно оценивая ситуацию, становится очевидно, что для обнаружения АДС было необходимо выполнение дуоденотомии. Однако отсутствие признаков кровотечения при ФГДС не позволило установить диагноз во время первой лапаротомии. Описанные выше хирургические вмешательства следует считать ошибочными.

\section{Заключение}

Вторичный АДС является редким источником кишечного кровотечения, которое зачастую бывает фатальным [Стяжкина и др., 2017; Simó Alari et al., 2017; Saito et al., 2019]. Ведущим методом диагностики АДС следует считать различные варианты ангиографии, однако приведенное наблюдение показывает, что инвазивная ангиография и МСКТ не всегда могут обнаружить прямой признак кровотечения - уклонение контрастированной крови. Прежде всего следует ориентироваться на клиническую картину. Эпизоды рецидивирующего массивного ЖКК при наличии в анамнезе протезирования аорты и отсутствие других очевидных причин свидетельствуют о наличии АДС. Шансов самостоятельного закрытия свища нет. Единственным способом лечения является оперативный. В представленном наблюдении даже ранняя операция (после первого геморрагического эпизода), скорее всего, оказалась бы безуспешной, учитывая выраженные адгезивные изменения в животе, интенсивный инфекционный процесс.

\section{Список литературы}

1. Андрейчук К.А., Сорока В.В., Андрейчук Н.Н. 2015. Редкие формы осложненных аневризм брюшной аорты. Кардиология и сердечно-сосудистая хирургия. 8 (4): 30-36. 
2. Батвинков Н.И., Горячев П.А., Василевский В.П., Кардис А.И., Цилиндзь А.Т., Труханов А.В. 2013. Аорто-энтеральная фистула как причина желудочно-кишечных кровотечений. Журнал Гродненского государственного медицинского университета, 3 (43): 105-107.

3. Костоглодова Н.С., Злобина А.И., Бровко С.В. 2016. Опыт оперативного лечения ложной аневризмы инфраренального отдела брюшной аорты, осложненной аорто-дуоденальной фистулой с развитием профузного желудочно-кишечного кровотечения. В кн.: Материалы Х Юбилейной Международной научно-практической конференции молодых ученыХ-медиков (Курск, 26-27 февраля 2016 г.). Курск, Изд-во КГМУ: 381-384.

4. Куликовский В.Ф., Карпачев А.А., Солошенко А.В., Ярош А.Л., Николаев С.Б., Коржова А.А., Гнашко А.В. 2017. Клинические случаи редких кровотечений из верхних отделов желудочно-кишечного тракта. Научные ведомости Белгородского государственного университета. Серия: Медицина. Фармация, 19 (268): 118-126.

5. Лебедев Н.В., Климов А.Е. 2010. Язвенные гастродуоденальные кровотечения. М.: Изд-во БИНОМ, 176.

6. Покровский А.В. 1979. Заболевание аорты и её ветвей. М., Медицина, 328.

7. Романович А.В., Хрыщанович В.Я., Кикоин Г.С., Турлюк Д.В. 2016. Случай успешной реконструкции аорто-бедренного сегмента нативным аортальным аллографтом при инфицировании синтетического протеза с формированием аорто-дуоденальной фистулы. Экстренная медицина, 5 (2): 302-308.

8. Руководство по неотложной хирургии органов брюшной полости. Под редакцией В.С. Савельева. М., Изд-во Триада-Х, 2005, 640.

9. Стяжкина С.Н., Климентов М.Н., Леонова А.Д., Мордвина А.Н., Насибова Ш.Х., Замараева Д.Д. 2017. Аорто-дуоденальная фистула (клинические наблюдения). Проблемы современной науки и образования, 14 (96): 108-111.

10. Трушникова Р.В., Муллахметова О.А., Зыков С.Ю. 2018. Спорадические случаи осложнений аневризмы аорты в практике врача-рентгенолога при выполнении КТ-аортографии. Российский электронный журнал лучевой диагностики, 8 (1): 202-207.

11. Colombi D., Bodini F.C., Sverzellati N., Morelli N., Capelli P., Michieletti E. 2018. A case of recurrent secondary aortoenteric fistula 4 months after surgery treated by endovascular coiling of the aortic stump and bilateral chimney stent grafts to renal arteries. Ann. Vasc. Surg, 59: 310. e1-310. e5. doi: 10.1016/j.avsg. 12.104 .

12. Lirici M.M., Tierno S.M., Giudice R., Coscarella C., Graziani M.G., Pogany G. 2019. Secondary aortoenteric fistula successfully treated with staged endovascular repair and duodenal resection without graft removal. Minim. Invasive. Ther. Allied. Technol., 8: 1-6. doi: 10.1080/13645706.2019.1581623.

13. Miyamoto K., Inaba M., Kojima T., Niguma T., Mimura T. 2016. Intra-aortic balloon occlusion (IABO) may be useful for the management of secondary aortoduodenal fistula (SADF): A case report. Int. J. Surg. Case. Rep., 25: 234-237. doi: 10.1016/j.ijscr.2016.06.010.

14. Saito H., Nishikawa Y., Akahira J.I., Yamaoka H., Okuzono T., Sawano T., Tsubokura M., Yamaya K. 2019. Secondary aortoenteric fistula possibly associated with continuous physical stimulation: a case report and review of the literature. J. Med. Case. Rep., 13 (1): 61. doi: 10.1186/s13256-019-2003-1.

15. Simó Alari F., Molina González E., Gutierrez I., Ahamdanech-Idrissi A. 2017. Secondary aortoduodenal fistula and the unrecognised herald bleed. Case Reports, 2017: bcr-2017-220186. doi: $10.1136 /$ bcr-2017-220186.

16. Šumskienė J., Šveikauskaitė E., Kondrackienė J., Kupčinskas L. 2016. Aorto-duodenal fistula: a rare but serious complication of gastrointestinal hemorrhage. A case report. Acta Med Litu, 23 (3): 165-168. doi: 10.6001/actamedica.v23i3.3380.

\section{References}

1. Andrejchuk K.A., Soroka V.V., Andrejchuk N.N. 2015. Redkie formy oslozhnennyh anevrizm brjushnoj aorty. Kardiologija i serdechno-sosudistaja hirurgija [Rare forms of complicated abdominal aortic aneurysms. Cardiology and cardiovascular surgery]. 8 (4): 30-36.

2. Batvinkov N.I., Gorjachev P.A., Vasilevskij V.P., Kardis A.I., Cilindz' A.T., Truhanov A.V. 2013. Aorto-jenteral'naja fistula kak prichina zheludochno-kishechnyh krovotechenij [Aorto-enteric fistula as the cause of gastro-enteric bleedings]. Zhurnal Grodnenskogo gosudarstvennogo medicinskogo universiteta, 3 (43): 105-107. 
3. Kostoglodova N.S., Zlobina A.I., Brovko S.V. 2016. Opyt operativnogo lechenija lozhnoj anevrizmy infrarenal'nogo otdela brjushnoj aorty, oslozhnennoj aorto-duodenal'noj fistuloj s razvitiem profuznogo zheludochno-kishechnogo krovotechenija [Experience of surgical treatment of a false aneurysm of the infrarenal abdominal aorta complicated by aorto-duodenal fistula with the development of profuse gastrointestinal bleeding]. V kn.: Materialy X Jubilejnoj Mezhdunarodnoj nauchno-prakticheskoj konferencii molodyh uchenyh-medikov (Kursk, 26-27 fevralja 2016 g.). Kursk, Izd-vo KGMU: 381-384.

4. Kulikovskij V.F., Karpachev A.A., Soloshenko A.V., Jarosh A.L., Nikolaev S.B., Korzhova A.A., Gnashko A.V. 2017. Klinicheskie sluchai redkih krovotechenij iz verhnih otdelov zheludochno-kishechnogo trakta [Clinical cases of rare bleeding of the upper sections of gastrointestinal tract]. Nauchnye vedomosti Belgorodskogo gosudarstvennogo universiteta. Serija: Medicina. Farmacija, 19 (268): 118-126.

5. Lebedev N.V., Klimov A.E. 2010. Jazvennye gastroduodenal'nye krovotechenija [Ulcerative gastroduodenal hemorrhages]. M.: Izd-vo BINOM, 176.

6. Pokrovskij A.V. 1979. Zabolevanie aorty i ejo vetvej [Disease of the aorta and its branches]. M., Medicina, 328.

7. Romanovich A.V., Hryshhanovich V.Ja., Kikoin G.S., Turljuk D.V. 2016. Sluchaj uspeshnoj rekonstrukcii aorto-bedrennogo segmenta nativnym aortal'nym allograftom pri inficirovanii sinteticheskogo proteza s formirovaniem aorto-duodenal'noj fistuly. Jekstrennaja medicina [A case of successful reconstruction of aorto-femoral segment using fresh aortic allograft in treatment of paraprostethic infection with formation of aorto-duodenal fistula]. 5 (2): 302-308.

8. Rukovodstvo po neotlozhnoj hirurgii organov brjushnoj polosti [Guidelines for emergency abdominal surgery]. Pod redakciej V.S. Savel'eva. M., Izd-vo Triada-H, 2005, 640.

9. Stjazhkina S.N., Klimentov M.N., Leonova A.D., Mordvina A.N., Nasibova Sh.H., Zamaraeva D.D. 2017. Aorto-duodenal'naja fistula (klinicheskie nabljudenija) [Aorta-duodenal fistula (case report)]. Problemy sovremennoj nauki i obrazovanija, 14 (96): 108-111.

10. Trushnikova R.V., Mullahmetova O.A., Zykov S.Ju. 2018. Sporadicheskie sluchai oslozhnenij anevrizmy aorty v praktike vracha-rentgenologa pri vypolnenii KT-aortografii [Sporadic cases of complications of an aortic aneurysm in the practice of the doctor - the radiologist when performing CTaortography]. Rossijskij jelektronnyj zhurnal luchevoj diagnostiki, 8 (1): 202-207.

11. Colombi D., Bodini F.C., Sverzellati N., Morelli N., Capelli P., Michieletti E. 2018. A case of recurrent secondary aortoenteric fistula 4 months after surgery treated by endovascular coiling of the aortic stump and bilateral chimney stent grafts to renal arteries. Ann. Vasc. Surg., 59: 310. e1-310. e5. doi: 10.1016/j.avsg. 12.104.

12. Lirici M.M., Tierno S.M., Giudice R., Coscarella C., Graziani M.G., Pogany G. 2019. Secondary aortoenteric fistula successfully treated with staged endovascular repair and duodenal resection without graft removal. Minim. Invasive. Ther. Allied. Technol., 8: 1-6. doi: 10.1080/13645706.2019.1581623.

13. Miyamoto K., Inaba M., Kojima T., Niguma T., Mimura T. 2016. Intra-aortic balloon occlusion (IABO) may be useful for the management of secondary aortoduodenal fistula (SADF): A case report. Int. J. Surg. Case Rep., 25: 234-237. doi: 10.1016/j.ijscr.2016.06.010.

14. Saito H., Nishikawa Y., Akahira J.I., Yamaoka H., Okuzono T., Sawano T., Tsubokura M., Yamaya K. 2019. Secondary aortoenteric fistula possibly associated with continuous physical stimulation: a case report and review of the literature. J. Med. Case. Rep., 13 (1): 61. doi: 10.1186/s13256-019-2003-1.

15. Simó Alari F., Molina González E., Gutierrez I., Ahamdanech-Idrissi A. 2017. Secondary aortoduodenal fistula and the unrecognised herald bleed. Case Reports, 2017: bcr-2017-220186. doi: 10.1136/bcr-2017-220186.

16. Šumskienė J., Šveikauskaitė E., Kondrackienẻ J., Kupčinskas L. 2016. Aorto-duodenal fistula: a rare but serious complication of gastrointestinal hemorrhage. A case report. Acta Med Litu, 23 (3): 165-168. doi: 10.6001/actamedica.v23i3.3380.

\section{Ссылка для цитирования статьи For citation}

Панасюк А.И., Кыштымов С.А., Иноземцев Е.О., Григорьев Е.Г 2020. Вторичный аортодуоденальный свищ. Рецидивирующее кровотечение. Трудности топической диагностики и лечебно-тактические ошибки (случай из клинической практики). Актуальные проблемы медицины, 43(1): 146-154. DOI

Panasyuk A.I., Kyshtymov S.A., Inozemcev E.O., Grigoryev E.G. 2020. Secondary aortoduodenal fistula. Recurrent bleeding. Difficulties of topical diagnosis and tactical errors (case from clinical practice). Challenges in Modern Medicine, 43(1): 146-154 (in Russian). DOI 